\title{
Line Spread Instrumentation for Propagation Measurements
}

\author{
Wade H. Bailey Jr., Captain, USAF \\ Air Force Weapons Laboratory/LRO \\ Kirtland AFB, NM 87117
}

\begin{abstract}
The Air Force Weapons Laboratory (AFWL) has been involved in the study of laser propagation from airborne platforms. A Line Spread Device (LSD) capable of yielding direct measure of a laser beam's Line Spread Function (LSF) was developed and employed in propagation tests conducted in a wind tunnel by AFWL to examine optimal acoustical suppression techniques for laser cavities exposed to simulated aircraft aerodynamic environments. Measurements were made on various aerodynamic fences and cavity air injection techniques that affect the LSF of a propagating laser. Using the quiescent tunnel as a control, the relative effect of each technique on laser beam quality was determined. The optical instrument employed enabled the comparison of relative beam intensity for each fence or mass injection. It was found that fence height had little effect on beam quality but fence porosity had a marked effect, i.e., $58 \%$ porosity alleviated cavity resonance and degraded the beam the least. Mass injection had little effect on the beam LSF. The use of a direct LSF measuring device proved to be a viable means of determining aerodynamic "seeing" qualities of flow fields. It could also be applied to static atmospheric "seeing" measurements through various gases and pollutants.
\end{abstract}

\section{Introduction}

An important aspect of the Air Force High Energy Laser Program is the examination of propagation effects associated with operating a laser from an airborne platform. This program studies in detail how the atmosphere would effect laser propagation, and, in particular, how the atmosphere would effect laser propagation in a dynamic situation, i.e., aerodynamic interference in the form of turbulent boundary layers, regions of separation, shock waves and other aerodynamic parameters associated with a possible airborne laser system. In conducting propagation measurements, large transonic wind tunnels were used to simulate conditions that could confront possible laser aircraft which may be employed in future weapons systems.

In order to quantify the optical performance of the numerous laser turret and aircraft models a device was required to measure laser beam quality as it was transmitted from these models into the surrounding flow field. Thus a device capable of direct measurement of the line spread function of coherent light evolved. The first generation Line Spread Device (LSD) was used in a wind tunnel test in April of 1975. An improved LSD instrument was planned for use in the follow-on wind tunnel test scheduled for July 1976. This latter series utilized the NASA Ames Research Center $6 \times 6$ foot transonic wind tunnel located at Moffett Field, California. The purpose of these tests was to develop cavity quieting techniques which permit efficient transmission of a laser beam from this exposed cavity into the surrounding flow field.

1453 received August 30,1977 . This paper was presented at the SPIE Seminar on Laser Technology for the Atmospheric Sciences, August 25-26, 1977, San Diego, California, and appears in SPIE Proceedings Volume 125 .

\section{Line Spread Device}

The Line Spread Device (LSD) was conceived as a simple instrument capable of measuring directly the optical quality of a laser beam after it has been propagated through an optically distorted medium. In theory, sweeping the focal spot of a coherent light source across a slit that is narrower than the focal spot, and detecting the intensity of the light as it traverses the slit yields an integrated slice of the Airy Disc. This is referred to as the Line Spread Function (LSF) of the beam and is illustrated in'Figure 1.

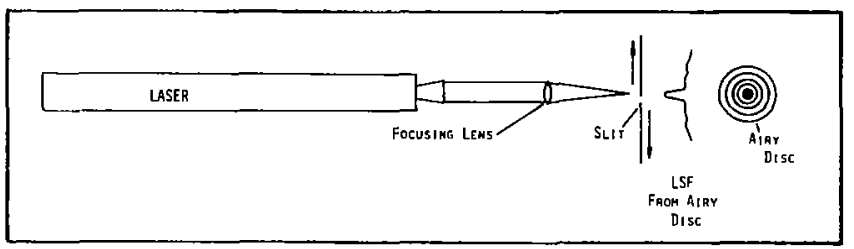

Figure 1. Principle of line spread function.

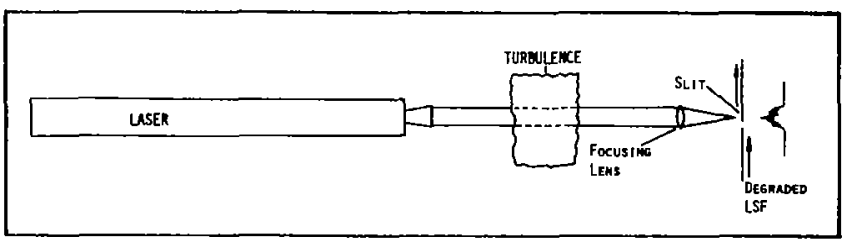

Figure 2. Output of optical disturbances.

Distortion of the line spread function occurs when the beam encounters an optically distorted medium, e.g., turbulence (see Figure 2).' A measure of beam degradation can be made by comparing the undistorted LSF to the distorted LSF. Beam quality can then be expressed using various methods. The first method would be a simple intensity ratio determined directly from the LSF. A simple beam quality figure of merit is then the ratio of the intensity of a distorted LSF(I) to an undistorted LSF intensity $\left(I_{0}\right)$. A second method would be to take the undistorted LSF and distorted LSF together and perform a Fast Fourier Transform which would produce a Modulation Transfer Function (MTF) of each. Taking the volume under each MTF and ratioing them as in the intensity method, a Strehl ratio can be determined. Phase tilt information can also be determined from the distorted beam as the tilt can cause lateral displacement of the LSF from a predetermined center line.

The line spread device used in the July 1976 wind tunnel test was relatively simple in construction and was composed entirely of off-the-shelf equipment. The optical components utilized in the LSD were of the highest quality. Figure 3 details the LSD as configured for the test. A 50 milliwatt helium-neon laser, Spectra-Physics model 125/250, was the source laser. A Spectra-Physics 2.5 centimeter collimator containing a microscope objective lens and a pinhole was used to spatially filter 


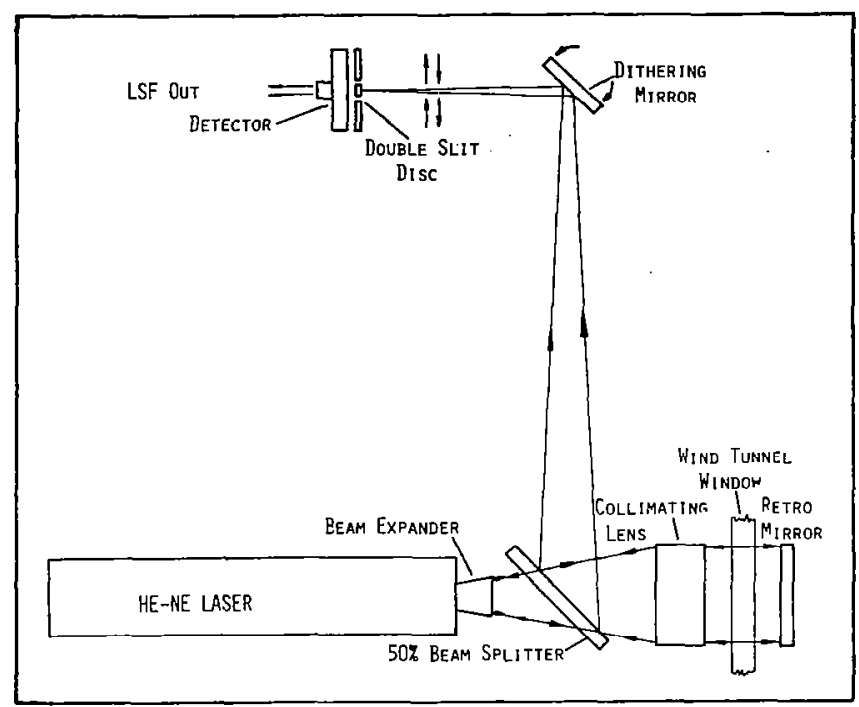

Figure 3. Line spread device conflguration.

the beam. The beam exited the pinhole and expanded through a $7.6 \times 5 \times 1.5$ centimeter $50 \%$ beam splitter for $6328 \AA$ wavelength light. The beam continued its expansion until it encountered a $10.2 \mathrm{~cm}$ lens. This lens was the compound collimating lens of a Tropel Model 280 laser collimator. The laser beam now collimated at $10.2 \mathrm{~cm}$ passed through an $11 \mathrm{~cm}$ diameter iris capable of stopping down to $0.5 \mathrm{~cm}$ diameter. The collimated beam then traversed the flow field being studied. A retromirror immersed in the flow field returned the beam back along its original path. Results from the wind tunnel test of April 1975 indicated that when the laser beam was allowed to pass completely across the six-foot test section of the wind tunnel and sampled on the opposite side of the tunnel, the inherent optical degradation of the wind tunnel flow overshadowed any model-induced degradation. The placement of a retro-mirror in the flow shortened the beam path in the wind tunnel and thus permitted the effects of the various models to be discerned.

The beam returned from the retro-mirror, impinged the beam splitter, and was turned towards another mirror. This mirror was dithering at $60 \mathrm{Hertz}$ and was controlled in amplitude by a General Scanning Model AX-100 motor control. Frequency was controlled by a Wavetek model 144 sweep generator. The dither mirror was oriented so as to turn the beam $90^{\circ}$ and sweep the focused beam in a back-and-forth motion across a pair of $2 \mathrm{mi}-$ cron slits separated by 400 microns. The disc containing the slits was positioned directly over a photovoltaic detector which measured the line spread function of the laser beam. The complete line spread device as shown in Figure 3 was enclosed in a cardboard housing. A cardboard tube was constructed to enclose the beam as it exited the LSD housing until it entered the wind tunnel. The enclosures were employed to minimize the effects of the ambient room turbulence on the measurements.

\section{LSF Data Acquisition System}

As the focused beam was swept across the slits, the beam intensity was detected by a United Technology photovoltaic PIN-10 detector. The frequency response of the detector was enhanced by a bias circuit. The signal then went through a PAR amplifier Model 110 via triaxial cabling. The amplified signal was monitored on an oscilloscope which served to control signal level into the tape recorder. When proper voltage levels were achieved the signal was placed on a Bell and Howell 14 track tape recorder Model M-14G. IRIG B time was also recorded from a Datametrics time code generator.

\section{Experimental Setup}

The experimental objective was to determine the optically degrading effect on a laser beam that might occur as it propagated from an open cavity on an aircraft in flight. Figure 4 shows the

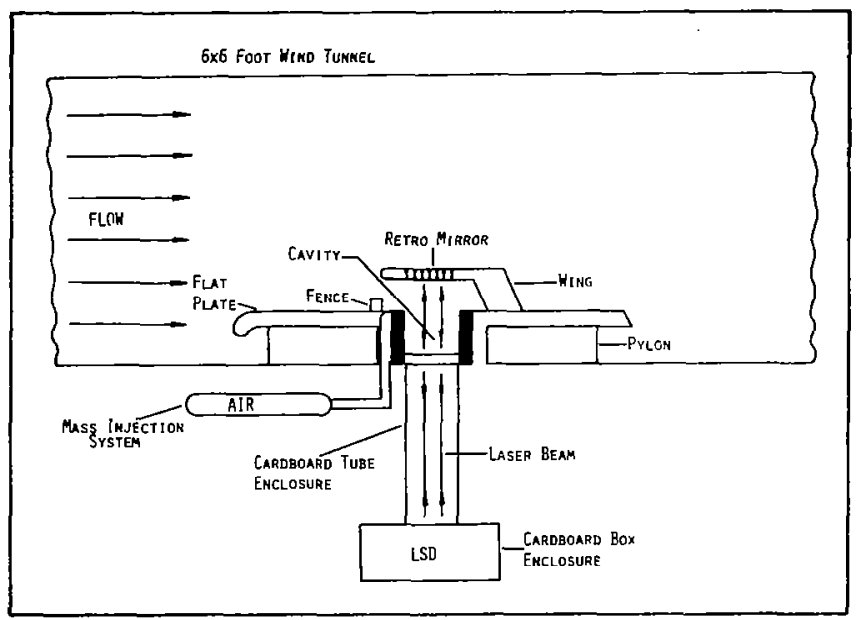

Figure 4. Top view of wind tunnel model configuration.

experimental setup. A splitter plate ( $3 \mathrm{~m} \times 1.2 \mathrm{~m}$ ) was mounted on a 25 centimeter pylon and placed on the side wall of the NASA Ames $6 \times 6$ foot transonic wind tunnel test section. A 6 inch cubical cavity was set into this plate. The bottom of the cavity (towards the wall) was constructed of high optical quality quartz. A "wing" was mounted on the plate to provide a mount for a return mirror which was 12 inches from the surface of the plate. Thus, an open cavity immersed in a high speed flow from which a laser could be propagated was simulated in the wind tunnel. This open cavity laser wind tunnel model was designed to simulate aircraft-induced effects.

The problems associated with airborne laser operation through an open cavity involve both acoustical and optical effects. The former are generated within the cavity and induce jitter within optical components. Optical effects are due mainly to the aerodynamic shear layer at the cavity exit. Methods to alleviate these effects were employed in this test. Metal bars referred to as "fences" were placed on the flat plate upstream of the cavity in an effort to suppress cavity acoustical noise. The fence heights ranged from $2.3 \mathrm{~cm}$ to $5.1 \mathrm{~cm}$, which were one and two times the existing boundary layer height on the flat plate. Each fence varied in porosity, i.e., holes werc drilled in the fences to permit mass flux through the fence. A method of injecting high pressure air into the cavity from the front wall was tried as an alternate means of quieting the cavity. As each technique was tested, the LSD made measurements of the LSF. Mach number was varied from $M=0.6$ to $M=0.89$ and Reynolds number was adjusted to either $R_{e}=2 \times 10^{6}$ per foot or $R_{e}=$ $3 \times 10^{6}$ per foot. These aerodynamic parameters were varied for each cavity suppression technique tested. Table 1 presents a listing of techniques and parameters tested with the LSD in this experiment for which an LSF was obtained.

The procedure in obtaining the LSF was first to calibrate the LSF signal for apertures ranging from $2 \mathrm{~cm}$ to $8 \mathrm{~cm}$ at one centimeter intervals. These calibrations were accomplished with the wind tunnel sealed and no air flowing. All calibrations were recorded on analog tape. Gain settings of the oscilloscopes and amplifiers were noted for each aperture calibration. An example of a two centimeter calibration can be seen in Figure 5. At the completion of the calibration series the wind tunnel was activated and allowed to stabilize at a particular Mach number and Reynolds number. At each aerodynamic condition, LSF data was recorded on tape. As each aperture was scanned during the 
Table 1. Wind Tunnel Model Configurations and the Aerodynamic Parametors Varied on Each

\begin{tabular}{|c|c|c|c|}
\hline Model Configuration & $\begin{array}{l}\text { Aperture } \\
\text { Scan }(\mathrm{cm})\end{array}$ & Reynolds No. & Mach Number \\
\hline $\begin{array}{l}\text { Flat plate, no } \\
\text { cavity }\end{array}$ & $2,4,6,8$ & $\begin{array}{l}2 \times 10^{6} \\
3 \times 10^{6}\end{array}$ & $.6, .7, .8, .9$ \\
\hline $\begin{array}{l}\text { Flat plate, cavity, } \\
\text { fence: } 5.1 \mathrm{~cm} \text { high } \\
49 \% \text { porosity }\end{array}$ & $2,4,6,8$ & $\begin{array}{l}2 \times 10^{6} \\
3 \times 10^{6}\end{array}$ & $.6, .7, .8, .9$ \\
\hline $\begin{array}{l}\text { Flat plate, cavity, } \\
\text { porous upstream } \\
\text { cavity wall }\end{array}$ & $2,5,8$ & $3 \times 10^{6}$ & $.6, .7, .8, .9$ \\
\hline $\begin{array}{l}\text { Flat plate, cavity, } \\
\text { fence: } 2.3 \mathrm{~cm} \text { high } \\
58 \% \text { porosity, porous } \\
\text { upstream cavity wall, } \\
\text { air injection into cavity }\end{array}$ & 5,7 & $3 \times 10^{6}$ & $.7, .9$ \\
\hline $\begin{array}{l}\text { Flat plate, cavity, } \\
\text { fence: } 2.3 \mathrm{~cm} \text { high } \\
58 \% \text { porosity }\end{array}$ & $2,5,7$ & $\begin{array}{l}2 \times 10^{6} \\
3 \times 10^{6}\end{array}$ & $.6, .7, .9$ \\
\hline $\begin{array}{l}\text { Flat plate, cavity, } \\
\text { fence: } 4.6 \mathrm{~cm} \text { high } \\
58 \% \text { porosity }\end{array}$ & $2,5,7$ & $\begin{array}{l}2 \times 10^{6} \\
3 \times 10^{6}\end{array}$ & $.6, .7, .9$ \\
\hline
\end{tabular}

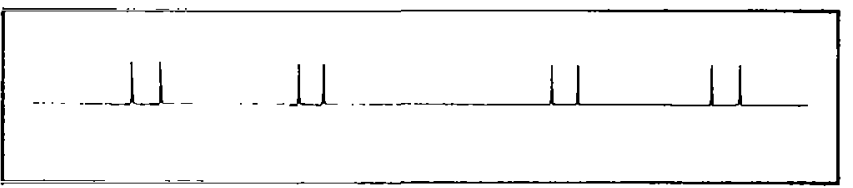

Figure 5. Example of LSF calibration $2 \mathrm{~cm}$ aperture.

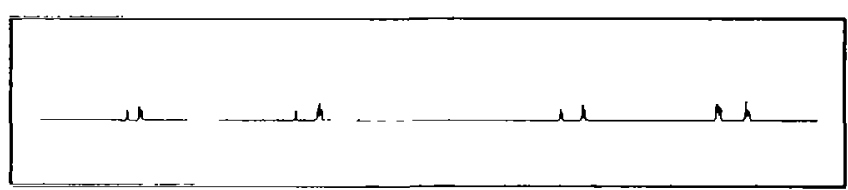

Figure 6. Example of LSF after traversing an optically degraded flow field $5 \mathrm{~cm}$ aperture.

run, the oscilloscope and amplifier settings were repeated as dictated by the calibration runs. The LSF of a degraded bearn is shown in Figure 6 after it experienced an active flow field. When the wind tunnel was stopped for a configuration change, an additional calibration run was accomplished.

\section{Discussion}

The reduction of the data was to utilize the recorded line spread function in conjunction with a Tektronic DPO 1221 system. Strehl ratios were calculated by employing programs developed for the Tektronix system. In particular, 10 to 100 distorted LSFs for a test run were centered according to area in relation to a predetermined coordinate system thus producing an average LSF. (It is known that by centering the LSFs and averaging, phase tilt information is lost; however, due to the vibrational environment of a wind tunnel, jitter of the beam cannot be separated from the motion of the optics or jitter induced by the flow field.) With the average LSF, a Fast Fourier Transfer can be performed using the Tektronix to produce a modulation transfer function (MTF) of the beam. By comparing the peak MTF of the calibration signal for a particular aperture and the MTF of the degraded signal, one can obtain a Strehl ratio. This data reduction has merits because it permits comparison of this data to other data taken on previous tests by other methods. A quick and simple form of data reduction can be applied by taking the average peak intensity of the LSFs and making a ratio of the calibration intensity, i.e., $\mathrm{I} / \mathrm{I}_{\text {cal }}$.

\section{Results}

During the experiment it was discovered that jitter due to the vibrating optics, especially the retro-mirror in the wind tunnel, was introduced into the measurements thereby spreading the beam but not affecting peak intensity. The only method viable for use in data reduction was an intensity ratio of averaged test points to its corresponding calibration run. The data tape was placed on strip charts for each configuration tested. The calibration runs were also placed on strip charts. Samples of these are shown in Figures 5 and 6. From the strip charts an average peak intensity for each case was determined by using a Hewlett-Packard plot package. An average intensity of each case was tabulated using $40 \mathrm{LSFs}$ and compared to the calibration case. These intensity ratios for each cavity suppression technique were plotted and trends were scrutinized to determine the technique that degraded the beam the least.' Figures 7 through 10 show the re-

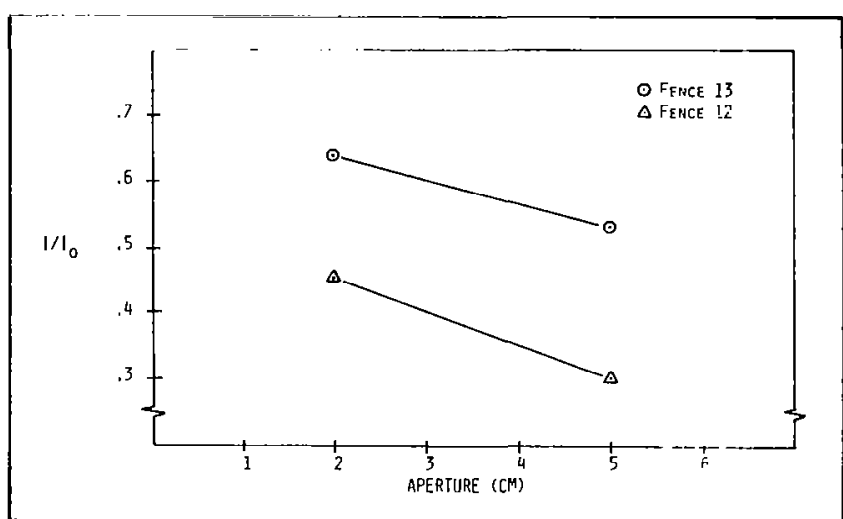

Figure 7. Comparison of two fances at $M-0.9$ and $R_{e}=2 \times 10^{6} / \mathrm{ft}$. Fence 13: $58 \%$ porosity and $2.2 \mathrm{~cm}$ in height. Fence 12: $58 \%$ porosity and $4.5 \mathrm{~cm}$ in height.

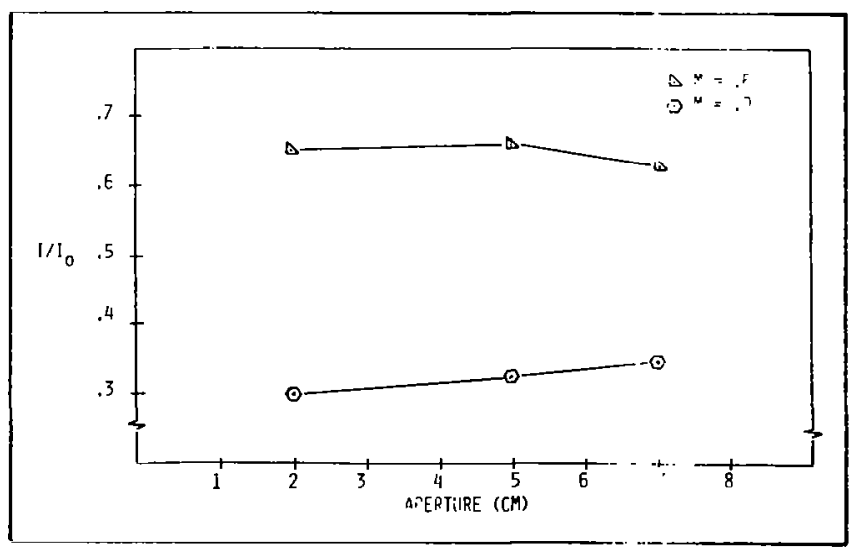

Figure 8. Comparison of Mach number dependence. Cavity model with Fence 13. $R_{e}=3 \times 10^{6} / \mathrm{ft}$.

sults in comparison form. From the trends in the LSF data it was determined that $2.2 \mathrm{~cm}, 58 \%$ porosity fence was the best optically and that mass injection had little effect on better "seeing." Reynolds number increase degraded optical seeing as well as an increase in Mach number. Since this was the first time an attempt to measure the optical quality of cavity noise 


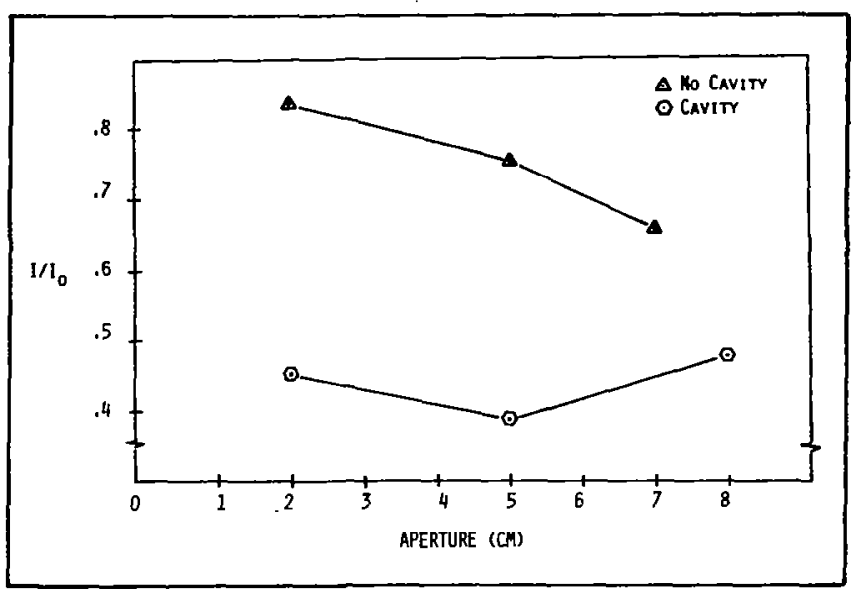

Figure 9. Comparison of flat plate with cavity and flat plate without covity. $M=0.7$ and $R_{0}=3 \times 10^{6} / \mathrm{ft}$.

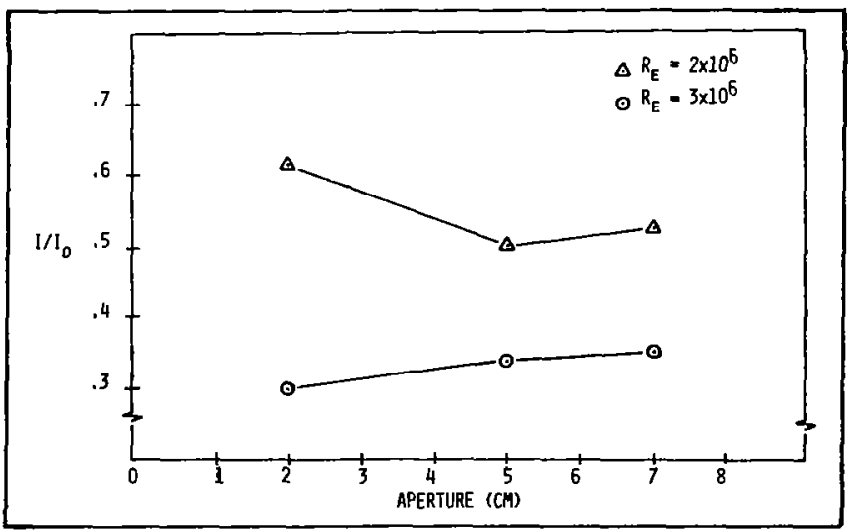

Figure 10. Comparison of Reynolds number dependence. Cavlty modet with Fence 13. $M=0.7$.

suppression techniques was made, no earlier results are available for comparison.

\section{Conclusion}

It can be concluded from the data results shown in Figures 7 through 10 that the line spread device is an instrument capable of direct measurements of light transmission quality through various atmospheric media. The LSD configuration employed introduced errors through optical component jitter caused by the severe wind tunnel environment. The component jitter may explain anomalies in the graph, e.g., optical "seeing" improving with increased aperture. Another source of error may lie in quirks of wind tunnel operation, in that certain tunnel conditions could alter the optical qualities of the flow. The "wing" that housed the retro-mirror produced a Venturi effect that was not anticipated. This effect may have "smoothed" the flow at high Mach numbers. The experiment as performed, and the LSD as configured, produced useful information pertinent to design of airborne laser optics.

\section{Recommendations}

The data as acquired by the LSD are applicable to many other areas of atmospheric transmission measurements to be made in the visible. The "seeing" quality of various gases and pollutants can be determined utilizing a line spread device. Additional work can be seen for this device and an improved model is being designed and fabricated by the author at this time. In order to alleviate the jitter experienced by the LSD in the severe vibrational environment of the wind tunnel, the new design will make use of a high speed rotating mirror. The high speed rotation will freeze any optical component jitter and maintain the integrity of the necessary constant velocity across the slit detector.

\section{Acknowledgments}

The author is deeply indebted to Major Keith G. Gilbert and Captain Richard $J$. Cook for their assistance in all aspects of the experimental work and theoretical considerations. Thanks is also due to Staff Sergeant Mark A. Cook for his unparalleled help in actual data acquisition. The author would like to express his gratitude to his wife, Cathy, Dr. David Depatie, and Airman Felix Ternate. Acknowledgment also is directed to MIT Lincoln Laboratory for use of unpublished MTF data contair,ed in D. Kelsall memo dated 26 April 1977.

\section{References}

1. AFWL TR 76-271, "Airborne Laser Laboratory Cycle II: Low-Power Experiments."

2. Meyer-Arendt, J. R., Introduction to Modern and Classical Optics, Prentice Hall, New Jersey, 1972. 